\title{
Malware Detection using Sub-Signatures and Machine Learning Technique
}

\author{
Ban Khammas \\ Collage of Information Engineering, University of Al-Nahrain \\ Iraq \\ banm1979@yahoo.com
}

\begin{abstract}
Malware is a major computer security concern as many computing systems are connected to the Internet. The number of malware has increased over the years and new malware has emerged, where new variants are capable of evading conventional system detection through obfuscations. One of the promising methods used to detect malware is machine learning (ML) techniques. This work presents a static malware detection system using n-gram and machine learning techniques, using known malware subsignatures to reduce large feature search spaces, which are generated due to n-gram feature extraction methods. The feature space directly affects the performance and the detection accuracy of malware ML classifiers. Analysis of multiple feature selection methods to minimize the number of features and analysis of multiple ML classifiers are also presented to improve the malware detection accuracy. The results show that analyzing n-gram with Snort sub-signature features using machine learning give good malware detection accuracy of more than $99.78 \%$ and zero FPR when 4-gram features are used for most of the verified ML classifiers.
\end{abstract}

Keywords: Machine Learning, Malware Detection, N-gram, Snort Signatures

Received: 23 February 2018, Revised 28 March 2018, Accepted 5 April 2018

DOI: $10.6025 / \mathrm{jisr} / 2018 / 9 / 3 / 96-106$

(C) 2018 DLINE. All Rights Reserved

\section{Introduction}

The rapid development in computers and networks communications led to exponential growth in internet users. This growth is accompanied with great challenges in network security. One of these challenges is the spread of malware all over the world [1]. According to the McAfee report [2], about an average of 100,000 new malware samples, appears every day. Malware has been responsible for major damage to the computing system in public institutions, including banks and companies, as well as personal computers. Machine learning techniques were recently proposed to detect malware. Machine learning generally focuses on finding connections in data and analysing the procedure for mining such relations. Numerous machine learning algorithms [3]-

$96 \quad$ Journal of Information Security Research Volume 9 Number 3 September 2018 
[7] have been proposed to detect malware. However, most of the proposed methods still cannot detect new and mutated malware with a high accuracy and a low false positive (FP), at the same time. It is very necessary to develop new detection techniques to stop the spread of malware as much as possible.

This paper presents a static malware detection method using Snort sub-signatures (n-gram signature) as features for machine learning classification, to detect malware directly from the file binaries. This work makes a comparison to find, the best feature selection method to minimize the ngram features space and the best ML classifier that can detect malware by enhancing the work [5], [7] using SNORT subsignatures to get high malware detection accuracy. Our aim is to produce a simple method that can be implemented in the host-based and network-based application layer to detect the malware accurately with the minimum feature space selected to detect malware. This paper is organized as follows: Section 2 reviews related works to support our hypothesis. Section 3 describes our methodology to detect malware in a host-based setup. Section 4 explains the experimental setup and datasets that have been used in this paper and reprocesses the datasets. Section 5 shows the experimental results. Finally, we conclude the paper in Section 6 and summarize the findings of this study.

\section{Related Work}

Malware is malicious software that has the ability to exploit vulnerabilities in the operating system and computer applications. Certain malware such as worms spread quickly through the network without user intervention. The loss due to malware is reported to be more than $\$ 10$ billion every year and increasing [8]. This type of attack replicates itself in the victim host and deploys a network to propagate to other hosts. Some types of malware are Trojan horses, virus, and worm [5]. Trojan Horses is a malicious program, which has a maleficent code covered into an executable file. Viruses are a type of malicious program, which have the ability to copy its malicious code inside other executable files [9]. On the other hand, worms is a type of malicious program that has the ability to self-replicate [10], it does not need to copy its own code inside other files like viruses to propagate [11]. A worm spreads automatically whereas a virus requires user intervention to spread [12].

To detect malware, there are two techniques that are either in the form of dynamic analysis or static analysis. The dynamic analysis focuses on actions or behaviour of the malware at the run time by executing malware on an isolated system or a virtual machine, taking snapshots and studying its behaviour [13]. The static analysis focuses on a complete analysis of malware without the need to execute the malware [14].

It is impossible to understand and analyse all the malware variants manually, based on matching the known malware signatures [15]. Therefore, machine learning (ML) has been proposed to detect malware [?], [3], [4]. ML techniques are used effectively in malware detection [?], [16]. There are many proposed research works to detect malware using ML and ngram such as [5]-[7], [13], [14], [17], [18]. The earliest work is by Kolter and Maloof [5], wherein they proposed a method to detect malware using ML to classify the malware executable files using extracted n-grams from malware byte code. They used the Boolean attribute to represent n-gram features. The result shows that the Boosted Decision Tree achieved an area under the curve (AUC) of about 0.996 after they select the features from all the n-gram features space, which is a huge feature space using IG. Robert et al. [14] proposed an extraction of n-grams from the file binaries and represent the features with their frequency instead of using the Boolean attribute to represent features. They also normalized these frequencies by dividing the number of the specific features by the maximum frequency in their study. They reported an accuracy of above $95 \%$ after they selected the features from all the $\mathrm{n}-$ gram features space.

In another work by the same authors, the representation of the binary code of a file by using n-grams was an approved promising strategy, especially for a large data set [7]. However, in the work by Santos et al. [6], they extract n-gram features from the opcode and have used ML to classify the files. They have represented the features by its frequency after they normalized it by dividing the number of specific features by the total number of features that appeared in the document. This representation of features is also used in the present study but has been used for the raw byte features, and not for the opcode features. Instead of using a single classifier to detect malware with n-gram feature extraction, the research works by [17], [18] combined multiple classifiers to detect malware. Liangboonprakong and Sornil [13] have extracted sequential n-gram pattern features instead of each feature, individually. However, some researchers have used the Snort signatures to detect attack and malware in the network after combining them with ML [19]-[21].

Similar to the idea used by [5], [7], our new proposed method uses the features extracted from raw byte represented by n-gram features from the binary files. However, it selects only those features that contain the known malware subsignature rules as the

Journal of Information Security Research Volume 9 Number 3 September 2018 
first stage filtering and combines it with ML to classify new malware.

\section{Proposed Malware Detection Method}

We propose a method for the intrusion detection system to detect the malware at the host level. The big picture of the proposed method is shown in the following Figure 1. The proposed method extracts n-gram features from the content of the file, and then filters the huge number of n-gram features. Snort sub-signature is used as a first stage filtering process and only the features that exist in Snort sub-signature which are different from the previous work [22] are selected. A second filter stage, the feature selection method has been used. A classifier is used to classify selected n-gram features based on the generated classifier model.

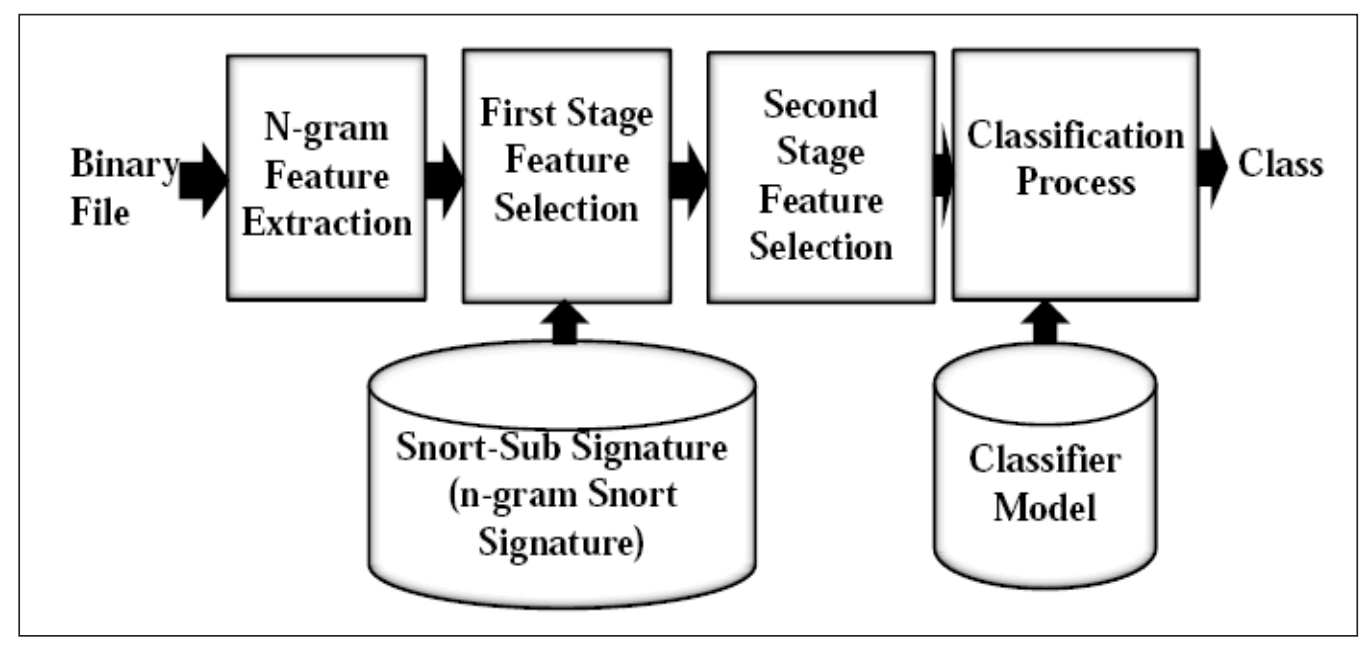

Figure 1. General architecture of the proposed method

\subsection{Feature Selection}

To select the most important sub-signature (n-gram features) that appear in malware files, different feature selection methods are tested. The feature selection methods that have been used in this work are Chi-square, CFsSubset, Principal Components, InfoGainAttribute, and GainRatioAttribute. Table I describes each of these types.

\subsection{Classification}

Classification is a process where the classifier learns from labeled data samples. The classifier is then tested for its classification accuracy using testing data samples. Each sample in the training set has one target value and several features. This overall process to classify unknown files as either malicious or benign is done by using ML methods. This process is divided into two stages: training and testing. The classifiers used in this work are SVM, J48, NB, IBK, and AdaBoost. The following Table II describes each type in brief.

\section{Experimental Setup}

The experimental setup for validating a proposed approach to detect malware using ML and malware sub-signature is explained in detail, in the following sections.

\subsection{Dataset}

First, the training data set must contain a labelled malware and normal dataset. The dataset contains normal executable files, which were collected from Windows 7, Windows XP operating systems, and Cygwin [23]. The total normal files are 1,971 files. We also collected general malware files that contain three different types of malware: Trojan, worm, and virus from VXHeavens [24]. They contain 550 files for Trojan, 550 files for worms, and 551 files for viruses (a total of 1,651 files). These types of malware are the most detected malware [5]. The total dataset for training are 3,622 files, which are the same number of files used in [5]. These malware and normal files are used for training phase of our methodology. For testing the classifier, a new dataset was collected, which contains 200 normal files and 257 malware files, (a total of 457 files).

98 Journal of Information Security Research Volume 9 Number 3 September 2018




\begin{tabular}{|l|l|}
\hline Feature Selection & \multicolumn{1}{c|}{ Definition } \\
\hline Chi-Square (ChiS) & $\begin{array}{l}\text { It measures the lack of independence between } \\
\text { a term and a category. Chi-Square is } \\
\text { described in detail, in [26]. }\end{array}$ \\
\hline CFsSubset (CFs) & $\begin{array}{l}\text { It detects linear dependencies between feature } \\
\text { and class, by evaluating the relationship } \\
\text { between feature and class. It selects the } \\
\text { features that have large correlation with the } \\
\text { class. More details can be found in [?], } \\
\text { [27]-[29]. }\end{array}$ \\
\hline $\begin{array}{l}\text { Principal } \\
\text { Components }\end{array}$ & $\begin{array}{l}\text { It is based on the conversion of a large } \\
\text { number of variables into a smaller number of } \\
\text { uncorrelated variables (Eigen vectors), by } \\
\text { finding a few orthogonal linear combinations } \\
\text { of the original variables with the largest } \\
\text { variance [30]. More details can be found in } \\
\text { [31],[32]. }\end{array}$ \\
\hline $\begin{array}{l}\text { InfoGainAttribute } \\
\text { (IG) }\end{array}$ & $\begin{array}{l}\text { It evaluates the feature according to the } \\
\text { measurement of the dependency between the } \\
\text { density of input variable and the density of } \\
\text { the target. More details can be found in [5], } \\
\text { [28]. }\end{array}$ \\
\hline GR) & $\begin{array}{l}\text { It is designed to overcome a bias in the } \\
\text { information gain by considering how the } \\
\text { feature splits the data. The details can be } \\
\text { found in [7]. }\end{array}$ \\
\hline
\end{tabular}

Table 1. Feature selection methods that are used in present study

\subsection{Dataset Preprocessing}

After collecting the dataset (normal and malware executable files), we use the hexdump utility to represent each executable by its hexadecimal codes and then generate n-grams. An Ngram of size four is used, based on the works by [13], [14], [25], wherein they showed that this number produced the best result for the classification performance.

\subsection{Evaluation Criteria}

To evaluate the result of our proposed method, and determine its precision and efficiency, we will measure the performance of each classifier in terms of accuracy, False Positive Rate (FPR), True Positive Rate (TPR), False Negative Rate (FNR), and True Negative Rate (TNR).

\section{Experimental Result Analysis}

The experiments were performed on Intel ${ }^{\circledR}$ core $^{\mathrm{TM}} \mathrm{i} 7-4710 \mathrm{HQ}, 250 \mathrm{GHZ}$ and $8 \mathrm{G}$ RAM with Linux Ubuntu 14.04, as the operating system. This work aims to analyse the ability to use Snort sub-signature and different feature selection methods with five different types of classifiers (IBK, SVM, Decision Tree (J48), NB, and AdaBoostIM) for n-gram features to detect malware using the WEKA tool [35]. 10- fold cross-validation was used to evaluate the approach and the methods, using the training dataset (3,622 files). Figure 2 shows the accuracy and AUC, also the FPR, FNR, TPR, and TNR are listed in Table III for the 10-fold cross validation of the training dataset.

AdaBoostIM, SVM, IBK, and J48 demonstrated the highest accuracy and relatively low false positive. NB provided a low Journal of Information Security Research Volume 9 Number 3 September 2018 


\begin{tabular}{|l|l|}
\hline Classifiers & Description \\
\hline J48 & $\begin{array}{l}\text { It is a Java implementation of C4.5. It divides } \\
\text { the training dataset space to local regions. It } \\
\text { computes the goodness of features to be the } \\
\text { root of the decision branch [33]. }\end{array}$ \\
\hline Naive Bayes (NB) & $\begin{array}{l}\text { It is a statistical classifier, which computes } \\
\text { the probability of sample to belong to a } \\
\text { specific class [21]. }\end{array}$ \\
\hline $\begin{array}{l}\text { Support Vector } \\
\text { Machine (SVM) }\end{array}$ & $\begin{array}{l}\text { It constructs a hyperplane or set of } \\
\text { hyperplanes in a high dimensional space. } \\
\text { SVM uses two key concepts to solve this } \\
\text { problem: large-margin separation and kernel } \\
\text { functions [34]. }\end{array}$ \\
\hline $\begin{array}{l}\text { Instance Based K } \\
\text { (IBK) }\end{array}$ & $\begin{array}{l}\text { This learning method, used by BIK, collects } \\
\text { the training samples or instances and tries to } \\
\text { find the (K) most similar instances in the } \\
\text { training sample, to the new testing file to } \\
\text { return the class prediction [5]. }\end{array}$ \\
\hline AdaBoostIM & $\begin{array}{l}\text { It uses ensemble learning from many } \\
\text { classifiers. The results of many classifiers are } \\
\text { combined together to represent a single } \\
\text { classification result [15]. }\end{array}$ \\
\hline
\end{tabular}

Table 2. Description of the classifiers that are used in the experiments

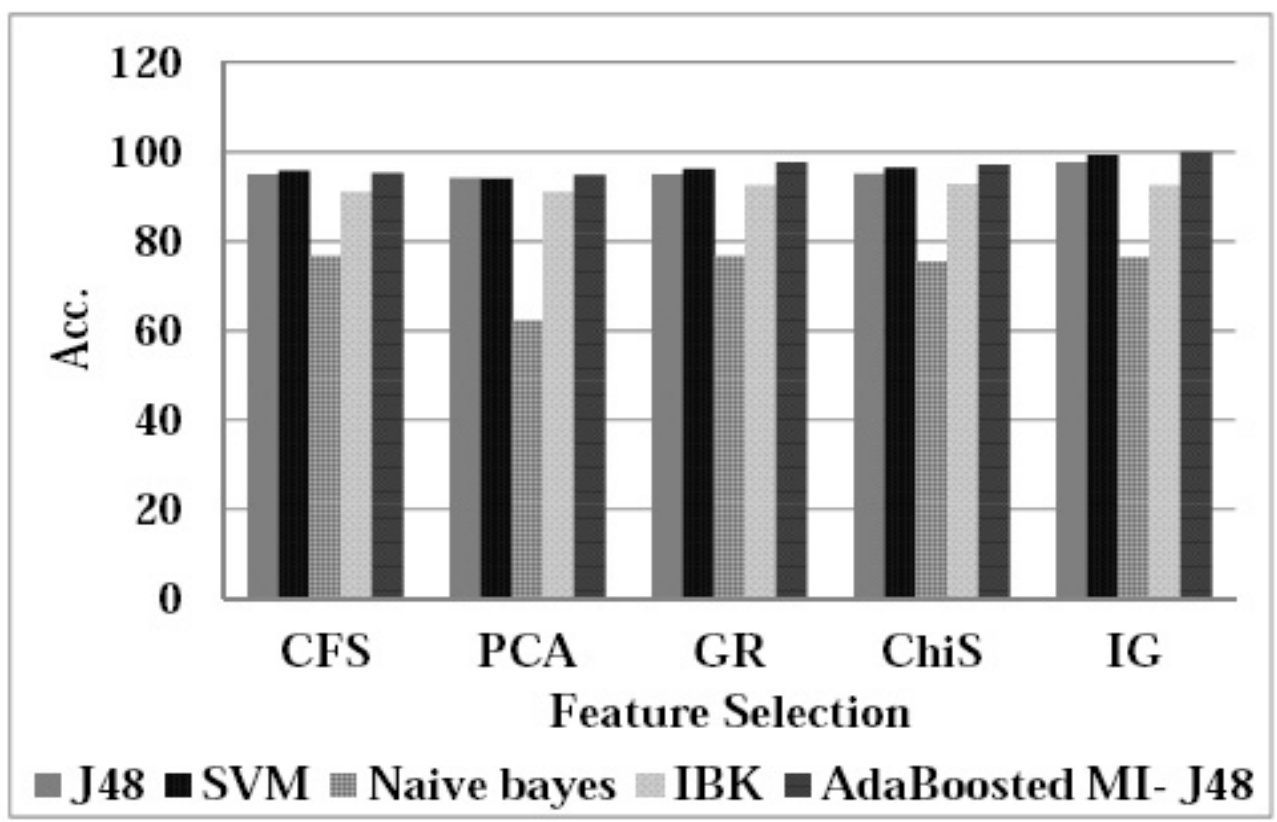

(a) Accuracy (Acc.) 


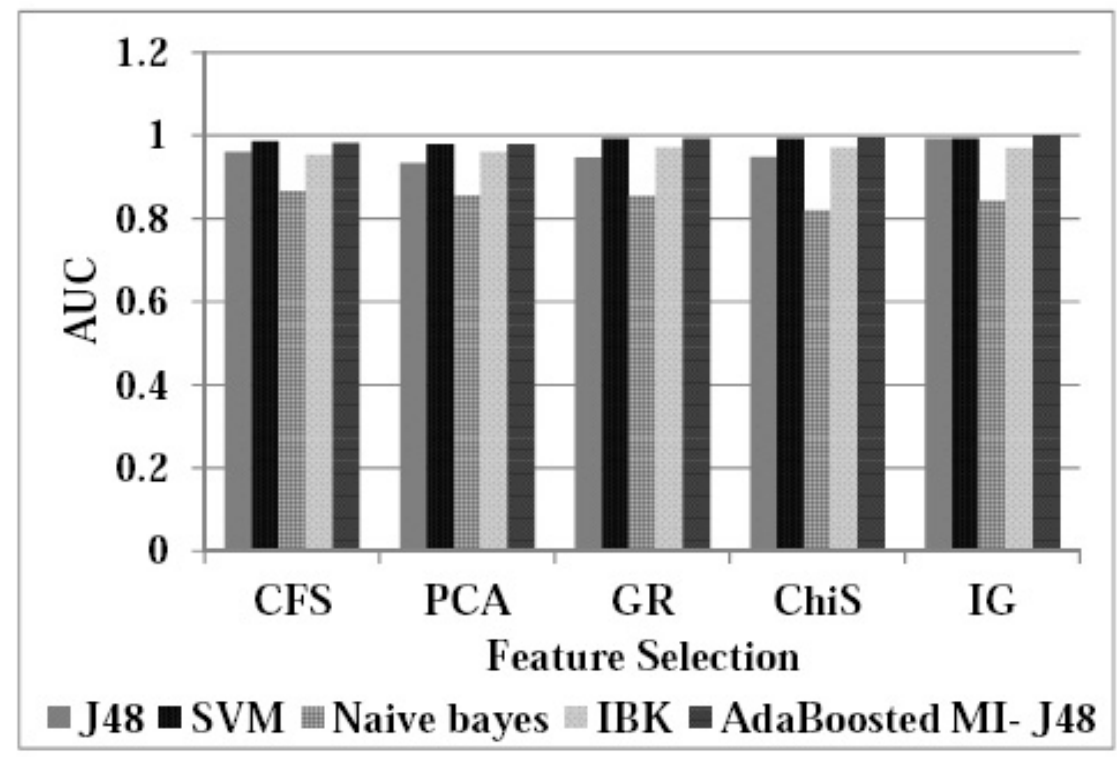

(b) Area under the curve (AUC)

Figure 2. ACC and AUC of different classifier using different feature selection method for 10-fold cross validation of the training dataset

accuracy and high false positive. For the features selected by a different feature selection method, CFsSubset, generates the least number of features. However, the accuracy of the classifiers is not very high when compared to other feature selection methods, especially, when a small number of features are used to detect a large number of new malware. The model that was built from using the features that were selected by IG, has the best accuracy. Similarly, the Chi Square, Gain Ratio Attribute, and PCA also gave it a good accuracy. The above result shows that AdaBoostMI and SVM have the highest accuracy and a high TPR and low FNR.

AdaBoostIM combined multiple classifiers in its structure, which used the ensemble method. Therefore, it needs a lot of time to build the model and a longer time for classifying the testing dataset. Figure 3 shows the comparison between the time to build the model of the training dataset for both classifiers.

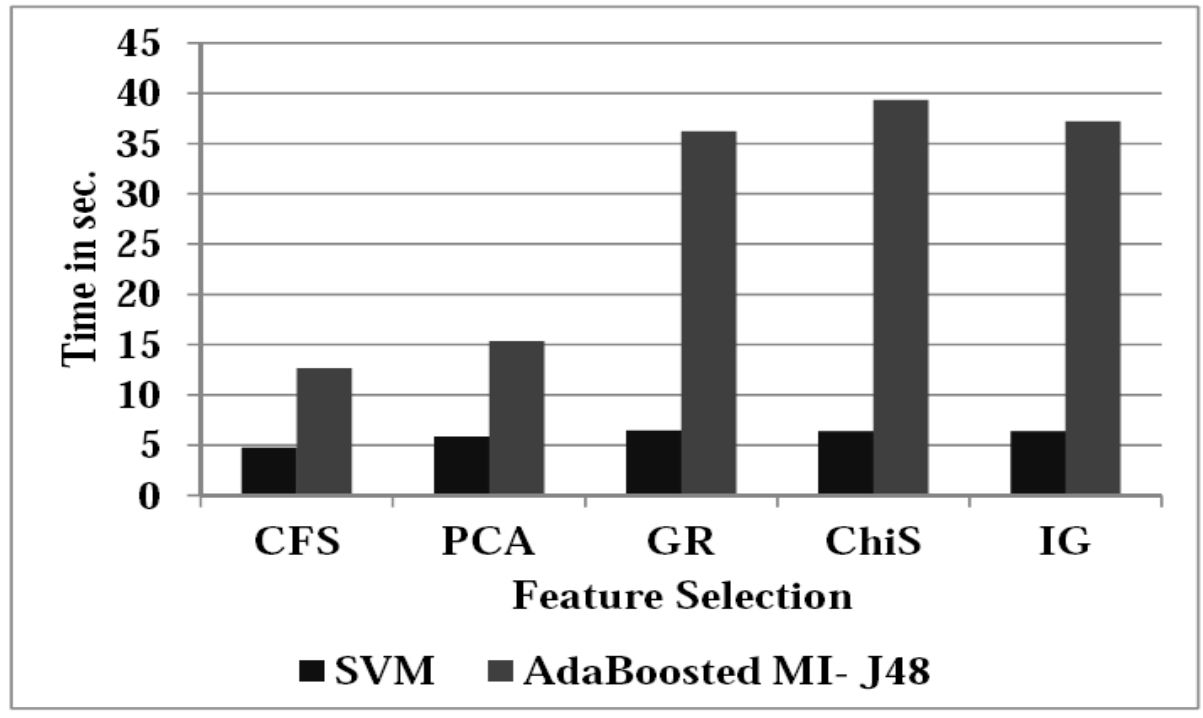

Figure 3. Time in sec. to build model of AdaBoosted MI-J48 and time to build model of SVM classifier using different feature selection method 


\begin{tabular}{|c|c|c|c|c|c|}
\hline \multirow{2}{*}{ Classifier } & \multicolumn{5}{|c|}{ Feature Selection } \\
\hline & CFS & PCA & GR & CHiS & IG \\
\hline $\mathrm{J} 48$ & $\begin{array}{l}\mathrm{FNR}=0.044 \\
\mathrm{FPR}=0.056 \\
\mathrm{TNR}=0.944 \\
\mathrm{TPR}=0.956\end{array}$ & $\begin{array}{l}\mathrm{FNR}=0.042 \\
\mathrm{FPR}=0.074 \\
\mathrm{TNR}=0.926 \\
\mathrm{TPR}=0.958\end{array}$ & $\begin{array}{l}\mathrm{FNR}=0.050 \\
\mathrm{FPR}=0.052 \\
\mathrm{TNR}=0.948 \\
\mathrm{TPR}=0.950\end{array}$ & $\begin{array}{l}\mathrm{FNR}=0.049 \\
\mathrm{FPR}=0.051 \\
\mathrm{TNR}=0.949 \\
\mathrm{TPR}=0.951\end{array}$ & $\begin{array}{l}\mathrm{FNR}=0.035 \\
\mathrm{FPR}=0.026 \\
\mathrm{TNR}=0.974 \\
\mathrm{TPR}=0.965\end{array}$ \\
\hline SVM & $\begin{array}{l}\mathrm{FNR}=0.053 \\
\mathrm{FPR}=0.035 \\
\mathrm{TNR}=0.965 \\
\mathrm{TPR}=0.947\end{array}$ & $\begin{array}{l}\mathrm{FNR}=0.073 \\
\mathrm{FPR}=0.048 \\
\mathrm{TNR}=0.952 \\
\mathrm{TPR}=0.927\end{array}$ & $\begin{array}{l}\mathrm{FNR}=0.055 \\
\mathrm{FPR}=0.024 \\
\mathrm{TNR}=0.976 \\
\mathrm{TPR}=0.945\end{array}$ & $\begin{array}{l}\mathrm{FNR}=0.050 \\
\mathrm{FPR}=0.023 \\
\mathrm{TNR}=0.977 \\
\mathrm{TPR}=0.950\end{array}$ & $\begin{array}{l}\mathrm{FNR}=0.001 \\
\mathrm{FPR}=0.013 \\
\mathrm{TNR}=0.987 \\
\mathrm{TPR}=0.999\end{array}$ \\
\hline $\begin{array}{l}\text { Naive } \\
\text { Bayes }\end{array}$ & $\begin{array}{l}\mathrm{FNR}=0.374 \\
\mathrm{FPR}=0.115 \\
\mathrm{TNR}=0.885 \\
\mathrm{TPR}=0.626\end{array}$ & $\begin{array}{l}\mathrm{FNR}=0.746 \\
\mathrm{FPR}=0.070 \\
\mathrm{TNR}=0.930 \\
\mathrm{TPR}=0.254\end{array}$ & $\begin{array}{l}\mathrm{FNR}=0.429 \\
\mathrm{FPR}=0.067 \\
\mathrm{TNR}=0.933 \\
\mathrm{TPR}=0.571\end{array}$ & $\begin{array}{l}\mathrm{FNR}=0.451 \\
\mathrm{FPR}=0.072 \\
\mathrm{TNR}=0.928 \\
\mathrm{TPR}=0.549\end{array}$ & $\begin{array}{l}\mathrm{FNR}=0.437 \\
\mathrm{FPR}=0.067 \\
\mathrm{TNR}=0.933 \\
\mathrm{TPR}=0.563\end{array}$ \\
\hline IBK & $\begin{array}{l}\mathrm{FNR}=0.102 \\
\mathrm{FPR}=0.080 \\
\mathrm{TNR}=0.920 \\
\mathrm{TPR}=0.898\end{array}$ & $\begin{array}{l}\mathrm{FNR}=0.133 \\
\mathrm{FPR}=0.052 \\
\mathrm{TNR}=0.948 \\
\mathrm{TPR}=0.867\end{array}$ & $\begin{array}{l}\mathrm{FNR}=0.118 \\
\mathrm{FPR}=0.039 \\
\mathrm{TNR}=0.961 \\
\mathrm{TPR}=0.882\end{array}$ & $\begin{array}{l}\mathrm{FNR}=0.114 \\
\mathrm{FPR}=0.038 \\
\mathrm{TNR}=0.960 \\
\mathrm{TPR}=0.886\end{array}$ & $\begin{array}{l}\mathrm{FNR}=0.116 \\
\mathrm{FPR}=0.039 \\
\mathrm{TNR}=0.961 \\
\mathrm{TPR}=0.884\end{array}$ \\
\hline $\begin{array}{l}\text { Ada- } \\
\text { Boost }\end{array}$ & $\begin{array}{l}\mathrm{FNR}=0.048 \\
\mathrm{FPR}=0.046 \\
\mathrm{TNR}=0.954 \\
\mathrm{TPR}=0.952\end{array}$ & $\begin{array}{l}\mathrm{FNR}=0.052 \\
\mathrm{FPR}=0.051 \\
\mathrm{TNR}=0.949 \\
\mathrm{TPR}=0.948\end{array}$ & $\begin{array}{l}\mathrm{FNR}=0.035 \\
\mathrm{FPR}=0.026 \\
\mathrm{TNR}=0.974 \\
\mathrm{TPR}=0.965\end{array}$ & $\begin{array}{l}\mathrm{FNR}=0.034 \\
\mathrm{FPR}=0.025 \\
\mathrm{TNR}=0.975 \\
\mathrm{TPR}=0.966\end{array}$ & $\begin{array}{l}\mathrm{FNR}=0 \\
\mathrm{FPR}=0 \\
\mathrm{TNR}=1 \\
\mathrm{TPR}=1\end{array}$ \\
\hline
\end{tabular}

Table 3. FPR, FNR, TPR, and TNR using 10-fold cross validation to the training dataset

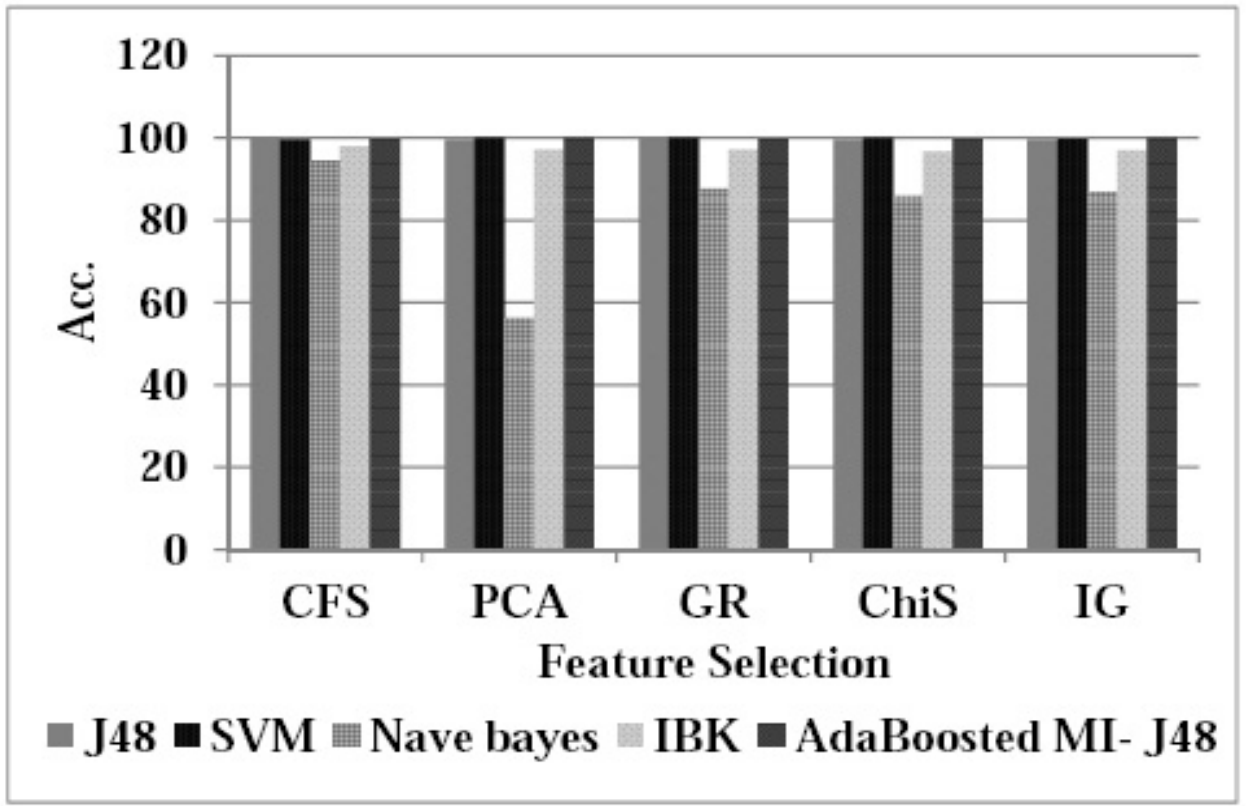

(a) Accuracy (Acc.). 


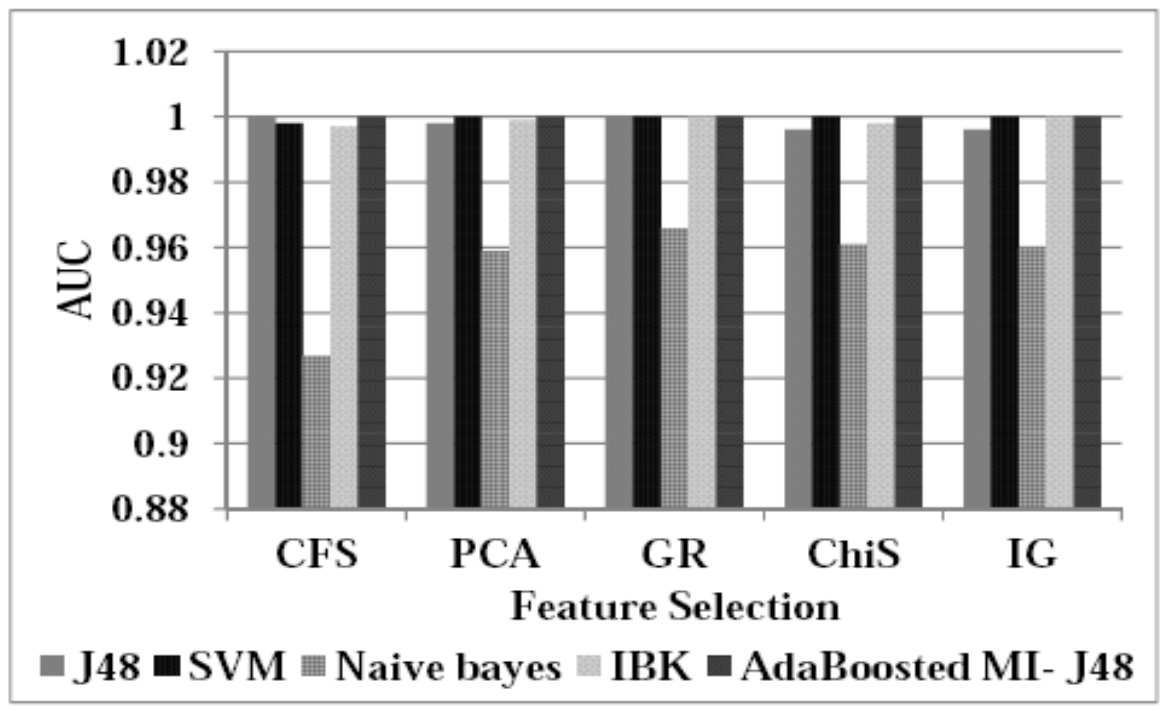

(b) Area under the curve (AUC).

Figure 4. Acc., AUC, FNR, FPR, TNR, and TPR of different classifier using different feature selection method for the testing dataset

\begin{tabular}{|c|c|c|c|c|c|}
\hline \multirow{2}{*}{ Classifier } & \multicolumn{5}{|c|}{ Feature Selection } \\
\hline & CFS & PCA & GR & CHiS & IG \\
\hline $\mathrm{J} 48$ & $\begin{array}{l}\mathrm{FNR}=0 \\
\mathrm{FPR}=0 \\
\mathrm{TNR}=1 \\
\mathrm{TPR}=1\end{array}$ & $\begin{array}{l}\mathrm{FNR}=0 \\
\mathrm{FPR}=0.01 \\
\mathrm{TNR}=0.99 \\
\mathrm{TPR}=1\end{array}$ & $\begin{array}{l}\mathrm{FNR}=0 \\
\mathrm{FPR}=0 \\
\mathrm{TNR}=1 \\
\mathrm{TPR}=1\end{array}$ & $\begin{array}{l}\mathrm{FNR}=0.004 \\
\mathrm{FPR}=0.005 \\
\mathrm{TNR}=0.995 \\
\mathrm{TPR}=0.996\end{array}$ & $\begin{array}{l}\mathrm{FNR}=0 \\
\mathrm{FPR}=0.01 \\
\mathrm{TNR}=0.99 \\
\mathrm{TPR}=1\end{array}$ \\
\hline SVM & $\begin{array}{l}\mathrm{FNR}=0.008 \\
\mathrm{FPR}=0 \\
\mathrm{TNR}=1 \\
\mathrm{TPR}=0.992\end{array}$ & $\begin{array}{l}\mathrm{FNR}=0 \\
\mathrm{FPR}=0 \\
\mathrm{TNR}=1 \\
\mathrm{TPR}=1\end{array}$ & $\begin{array}{l}\mathrm{FNR}=0 \\
\mathrm{FPR}=0 \\
\mathrm{TNR}=1 \\
\mathrm{TPR}=1\end{array}$ & $\begin{array}{l}\mathrm{FNR}=0 \\
\mathrm{FPR}=0 \\
\mathrm{TNR}=1 \\
\mathrm{TPR}=1\end{array}$ & $\begin{array}{l}\mathrm{FNR}=0.004 \\
\mathrm{FPR}=0 \\
\mathrm{TNR}=1 \\
\mathrm{TPR}=0.996\end{array}$ \\
\hline $\begin{array}{l}\text { Naive } \\
\text { Bayes }\end{array}$ & $\begin{array}{l}\mathrm{FNR}=0.058 \\
\mathrm{FPR}=0.05 \\
\mathrm{TNR}=0.95 \\
\mathrm{TPR}=0.942\end{array}$ & $\begin{array}{l}\mathrm{FNR}=0.74 \\
\mathrm{FPR}=0.04 \\
\mathrm{TNR}=0.95 \\
\mathrm{TPR}=0.25\end{array}$ & $\begin{array}{l}\mathrm{FNR}=0.179 \\
\mathrm{FPR}=0.05 \\
\mathrm{TNR}=0.95 \\
\mathrm{TPR}=0.821\end{array}$ & $\begin{array}{l}\mathrm{FNR}=0.21 \\
\mathrm{FPR}=0.05 \\
\mathrm{TNR}=0.95 \\
\mathrm{TPR}=0.79\end{array}$ & $\begin{array}{l}\mathrm{FNR}=0.21 \\
\mathrm{FPR}=0.05 \\
\mathrm{TNR}=0.95 \\
\mathrm{TPR}=0.79\end{array}$ \\
\hline IBK & $\begin{array}{l}\mathrm{FNR}=0.023 \\
\mathrm{FPR}=0.015 \\
\mathrm{TNR}=0.985 \\
\mathrm{TPR}=0.977\end{array}$ & $\begin{array}{l}\mathrm{FNR}=0.05 \\
\mathrm{FPR}=0 \\
\mathrm{TNR}=1 \\
\mathrm{TPR}=0.95\end{array}$ & $\begin{array}{l}\mathrm{FNR}=0.051 \\
\mathrm{FPR}=0 \\
\mathrm{TNR}=1 \\
\mathrm{TPR}=0.949\end{array}$ & $\begin{array}{l}\mathrm{FNR}=0.058 \\
\mathrm{FPR}=0 \\
\mathrm{TNR}=1 \\
\mathrm{TPR}=0.942\end{array}$ & $\begin{array}{l}\mathrm{FNR}=0.21 \\
\mathrm{FPR}=0.05 \\
\mathrm{TNR}=0.95 \\
\mathrm{TPR}=0.79\end{array}$ \\
\hline $\begin{array}{l}\text { Ada- } \\
\text { Boost }\end{array}$ & $\begin{array}{l}\mathrm{FNR}=0.004 \\
\mathrm{FPR}=0 \\
\mathrm{TNR}=1 \\
\mathrm{TPR}=0.996\end{array}$ & $\begin{array}{l}\mathrm{FNR}=0 \\
\mathrm{FPR}=0 \\
\mathrm{TNR}=1 \\
\mathrm{TPR}=1\end{array}$ & $\begin{array}{l}\mathrm{FNR}=0.004 \\
\mathrm{FPR}=0 \\
\mathrm{TNR}=1 \\
\mathrm{TPR}=0.996\end{array}$ & $\begin{array}{l}\mathrm{FNR}=0.004 \\
\mathrm{FPR}=0 \\
\mathrm{TNR}=1 \\
\mathrm{TPR}=0.996\end{array}$ & $\begin{array}{l}\mathrm{FNR}=0 \\
\mathrm{FPR}=0 \\
\mathrm{TNR}=1 \\
\mathrm{TPR}=1\end{array}$ \\
\hline
\end{tabular}

Table 4. FPR, FNR, TPR, and TNR of the testing dataset 


\begin{tabular}{|l|l|l|l|l|l|l|l|}
\hline $\begin{array}{l}\text { Existing } \\
\text { Work }\end{array}$ & $\begin{array}{l}\text { Feature } \\
\text { Type } \\
\text { Features }\end{array}$ & $\begin{array}{l}\text { No. } \\
\text { of }\end{array}$ & Ngram & Acc. or ROC & TPR & FPR & Description \\
\hline $\begin{array}{l}\text { Proposed } \\
\text { method } \\
\text { byte }\end{array}$ & 500 & 4 gram & $\begin{array}{l}100 \% \\
\text { (Acc.) }\end{array}$ & 1 & 0 & $\begin{array}{l}\text { Extract only } \\
\text { the features } \\
\text { that are } \\
\text { appeared in } \\
\text { Snort features, } \\
\text { most of the } \\
\text { classifiers do } \\
\text { well especially } \\
\text { (BDT) and } \\
\text { SVM. }\end{array}$ \\
\hline $\begin{array}{l}\text { Kolter \& } \\
\text { Maloof }\end{array}$ & $\begin{array}{l}\text { raw } \\
\text { byte }\end{array}$ & 500 & 4 gram & $\begin{array}{l}99.6 \% \\
\text { (ROC) }\end{array}$ & 0.98 & 0.05 & $\begin{array}{l}\text { Boosted } \\
\text { decision tree. }\end{array}$ \\
\hline $\begin{array}{l}\text { Moskovitch } \\
\text { et al. [7] }\end{array}$ & $\begin{array}{l}\text { raw } \\
\text { byte }\end{array}$ & 300 & 5 gram & $\begin{array}{l}94.9 \% \\
\text { (Acc.) }\end{array}$ & 0.99 & 0.04 & \begin{tabular}{l} 
NN \& DT. \\
\hline $\begin{array}{l}\text { Santos et } \\
\text { al. [36] }\end{array}$
\end{tabular} \\
$\begin{array}{l}\text { raw } \\
\text { byte }\end{array}$ & 1000 & 2 gram & $\begin{array}{l}88 \% \\
\text { (Acc.) } \\
88 \%\end{array}$ & 0.87 & 0.1 & $\begin{array}{l}\text { Local and } \\
\text { Global } \\
\text { Consistency } \\
\text { (LLGC). }\end{array}$ \\
\hline $\begin{array}{l}\text { Shabtai et } \\
\text { al. [37] }\end{array}$ & opcode & 300 & 2 gram & $\begin{array}{l}96 \% \\
\text { (Acc.) }\end{array}$ & 0.95 & 0.1 & $\begin{array}{l}\text { BDT \& } \\
\text { Random }\end{array}$ \\
\hline
\end{tabular}

Table 5. Comparison of the proposed system's results with several related report

For the testing dataset which contains 457 files (200 normal, and 257 malware), the result is shown in Figure 4 also the FPR, FNR, TPR, and TNR are listed in Table IV. The results that test the testing dataset after building the classifier from the training dataset show that AdaBoostIM, J48 and SVM have the lowest FPR and FNR and a high accuracy in detecting new malware using different feature selection methods.

From Table 2 and Table 4 which shows that AdaBoostIM is the best when using 10-fold cross validation in the training dataset and when used the training datadset to build the classifier model to test the testing dataset. Also J48, SVM, and IBK do well in different feature selection method to test the testing.

Table 5 depicts the comparison between some of the existing works with our method, to detect malware in the testing dataset using machine learning and n-gram.

\section{Conclusion}

In this paper, the focus lies on analyzing and measuring the detection accuracy of the ML classifier using n-gram Snort subsignatures to detect malware in host based. The proposed method aims to detect the new malware before or during the software installation by analyzing the binary files of the malware files. The result shows that using a very small number of Snort subsignature n-gram features (500 features only) will minimize the searching space of n-gram features, and still give a very high accuracy and low FPR to detect malware files for most of the classifiers; especially for J48, AdaBoostIM, and SVM in both the 10fold cross-validation and in the testing dataset. For all future work, we will use this methodology to detect new malware in the network-base and measure the capability of this method to detect them in the network level.

104 Journal of Information Security Research Volume 9 Number 3 September 2018




\section{References}

[1] Misra, A., Verma, M., Sharma, A. (2014). Capturing the interplay between malware and anti-malware in a computer network, Applied Mathematics and Computation, 229, 340-349.

[2] 2013 infographic, the state of malware.

[3] O'Kane, P., Sezer, S., McLaughlin, K., Im, E. G. (2013). Svm training phase reduction using dataset feature filtering for malware detection," IEEE Transactions on Information Forensics and Security, 8(3) 500-509.

[4] Vinod, P., Laxmi, V., Gaur, M. S. (2012). Reform: relevant features for malware analysis, $I n$ : $26^{\text {th }}$ International Conference on Advanced Information Networking and Applications Workshops (WAINA), 2012, p 738-744, IEEE.

[5] Kolter, J. Z., Maloof, M. A. (2006). Learning to detect and classify malicious executables in the wild," The Journal of Machine Learning Research, 7, p. 2721-2744.

[6] Santos, I., Brezo, F., Ugarte-Pedrero, X., Bringas, P. G. (2013). Opcode sequences as representation of executables for datamining-based unknown malware detection, Information Sciences, 231, p 64-82.

[7] Moskovitch, R., Stopel, D., Feher, C., Nissim, N., Japkowicz, N., Elovici, Y. (2009). Unknown malcode detection and the imbalance problem, Journal in Computer Virology, 5 (4). 295-308.

[8] Song, F., Touili, T. (2012). Efficient malware detection using modelchecking, In: FM 2012: Formal Methods, p. 418-433, Springer.

[9] Boyanov, P. K. (2013). A taxonomy of the cyber attacks, Journal Scientific \& Applied Research, 3.

[10] Li, P., Salour, M., Su, X. (2008). A survey of internet worm detection and containment, Communications Surveys \& Tutorials, IEEE, 10 (1) 20-35.

[11] Konakalla, A., Veeranki, B. (2013). Evolution of security attacks and security technology, International Journal of Computer Science and Mobile Computing, 2, p 270-276, November.

[12] Tian, R. (2011). An integrated malware detection and classification system, tech. rep., Deakin University.

[13] Liangboonprakong, C., Sornil, O. (2013). Classification of malware families based on n-grams sequential pattern features, In: $20138^{\text {th }}$ IEEE Conference on Industrial Electronics and Applications (ICIEA), p. 777-782, IEEE, 2013.

[14] Moskovitch, R., Stopel, D., Feher, C., Nissim, N., Elovici, Unknown malcode detection via text categorization and the imbalance problem," In: IEEE International Conference on Intelligence and Security Informatics, ISI 2008., p. 156-161, IEEE, 2008.

[15] LeDou, C., Lakhotia, A. (2015). Malware and machine learning, in Intelligent Methods for Cyber Warfare, p. 1-42, Springer.

[16] Shabtai, A., Moskovitch, R., Elovici, Y., Glezer, C. (2009). Detection of malicious code by applying machine learning classifiers on static features: A state-of-the-art survey, Information Security Technical Report, 14(1) 16-29.

[17] Zhang, B., Yin, J., Hao, J., Zhang, D., Wang, S. (2007). Malicious codes detection based on ensemble learning, In: Autonomic and Trusted Computing, p 468-477, Springer.

[18] Reddy, D. K. S., Pujari, A. K. (2006).N-gram analysis for computer virus detection, Journal in Computer Virology, 2 (3) 231239.

[19] de Sa Silva, L. A. C. Ferrari dos Santos, J. D. S. da Silva, Montes, A (2004). A neural network application for attack detection in computer networks, In: IEEE International Joint Conference on Neural Networks 2004, 2, p. 1569-1574, IEEE, .

[20]de Silva, L. 1., dos Santos, A. C. F., Mancilha, T. D., da Silva, J. D. S. S., Montes. A. (2008). Detecting attack signatures in the real network traffic with annida, Expert Systems with Applications, 34 (4) 2326-2333.

[21] Ismail, I., Marsono, M. N., Khammas, B. M., Nor. S. M. (2015). Incorporating known malware signatures to classify new malware variants in network traffic," International Journal of Network Management, 25 (6) 471-489.

[22] Khammas, B. M., Monemi, A., Joseph, S. B., Ismail, I., Mohd Nor, S., Marsono, M. N. (2015). Feature selection and machine learning classification for malware detection, Jurnal Teknologi, 77 (1) 234-250.

[23]Cygwin.

[24] Vx heavens.

Journal of Information Security Research Volume 9 Number 3 September 2018 
[25] Kolter, J. Z., Maloof, M. A. (2004). Learning to detect malicious executables in the wild, In: Proceedings of the $10^{\text {th }}$ ACM SIGKDD International Conference on Knowledge Discovery and Data Mining, 470-478, ACM.

[26] Zheng, Z., Wu, X., Srihari, R. Feature selection for text categorization on imbalanced data, ACM Sigkdd Explorations Newsletter, 6 (1) 80-89.

[27] Karegowda, A. G., Manjunath, Jayaram, M. (2010). “Comparative study of attribute selection using gain ratio and correlation based feature selection, International Journal of Information Technology and Knowledge Management, 2 (2) $271-277$.

[28] Guyon, I., Elisseeff, A. (2003). An introduction to variable and feature selection, The Journal of Machine Learning Research, $3,1157-1182$.

[29] Chandrashekar, G., Sahin, F. (2014). A survey on feature selection methods, Computers \& Electrical Engineering, 40 (1), 1628.

[30] Wang, W., Guan, X., Zhang, X. (2008). Processing of massive audit data streams for real-time anomaly intrusion detection, Computer Communications, 31 (1) 58-72.

[31] Wang, W., Zhang, X., Gombault, S. (2009). Constructing attribute weights from computer audit data for effective intrusion detection, Journal of Systems and Software, 82 (12) 1974-1981.

[32] Xu, X.,Wang, X. (2005). “An adaptive network intrusion detection method based on pca and support vector machines, In: Advanced Data Mining and Applications, p. 696-703, Springer.

[33] Boukhtouta, A., Mokhov, S. A., Lakhdari, N.-E., Debbabi, M., Paquet, J. (2015). Network malware classification comparison using dpi and flow packet headers, Journal of Computer Virology and Hacking Techniques, p. 1-32.

[34] Miao, Q., Liu, J., Cao, Y., Song, J. (2015). Malware detection using bilayer behavior abstraction and improved one-class support vector machines, International Journal of Information Security, 1-19.

[35] Weka.

[36] Santos, I., Nieves, J., Bringas, P. G. (2011). Semi-supervised learning for unknown malware detection, International Symposium on Distributed Computing and Artificial Intelligence, p. 415-422, Springer.

[37] Shabtai, A., Moskovitch, C., Feher, C., Dolev, S., Elovici, Y. (2012). Detecting unknown malicious code by applying classification techniques on opcode patterns," Security Informatics, 1 (1) 1-22. 